\title{
然
}

\section{EL TURISMO INTERIOR EN ESPAÑA ANTE LA CRISIS DE LA COVID-19}

El objetivo de este trabajo es detectar los cambios que se han producido en el turismo interior en España durante la pandemia de la COVID-19 y, sobre esa base, discutir el papel que puede desempeñar para facilitar la recuperación turística. La información estadística disponible confirma la importancia relativa que tiene el turismo interior y su potencial para compensar la caída de la demanda turística internacional. En el caso concreto de España, los datos sugieren que durante la pandemia se han sustituido los viajes internacionales por los nacionales y, más concretamente, por los realizados dentro de la propia comunidad autónoma. Respecto a las medidas implementadas por parte del Gobierno español se aprecia un sesgo a favor de las destinadas a facilitar la liquidez de las empresas, y se reconoce la capacidad que tiene el turismo interior para iniciar la recuperación.

Palabras clave: demanda turística, promoción turística.

Clasificación JEL: L83.

\section{Introducción}

La pandemia asociada a la COVID-19 ha tenido un impacto descomunal en la economía mundial y, especialmente, en el sector turístico (Škare et al., 2021). En lo que llevamos de siglo han existido otras crisis turísticas de carácter global. Los ataques terroristas del 11 de septiembre de 2001 (Blake y Sinclair, 2003), el síndrome respiratorio agudo severo (SARS) en 2003 (McKercher y Chon, 2004) y la Gran Recesión de 2007 (Pechlaner y Frehse, 2010) provocaron caídas de la demanda turística internacional. Sin

\footnotetext{
* Universidad de Málaga.

Versión de agosto de 2021.

DOI: https://doi.org/10.32796/bice.2021.3139.7285
}

embargo, la intensidad y la persistencia de la crisis turística causada por la COVID-19 no tiene precedentes. De hecho, la Organización Mundial del Turismo (OMT) ha calificado 2020 como el peor año en la historia del turismo (UNWTO, 2021).

Las peculiaridades de la reciente crisis turística se explican por la caída simultánea de la oferta y de la demanda. Por una parte, está suficientemente acreditado que el aumento de la percepción de riesgo ante determinados acontecimientos disminuye la demanda turística (Ritchie y Jiang, 2019). Es obvio que el riesgo al contagio hizo descender los viajes turísticos. Pero, por otro lado, y esto constituye un elemento novedoso, las medidas adoptadas para contener la expansión del virus colapsaron la $\triangleright$ 
oferta turística. En España, el Real Decreto 463/2020, de 14 de marzo, declaró el estado de alarma. Sobre esa base, la Orden SND/257/2020, de 19 de marzo, suspendió la apertura al público de los establecimientos de alojamiento turístico ubicados en cualquier parte del territorio nacional. Posteriormente, la Orden SND/399/2020, de 9 de mayo, y la Orden SND/414/2020, de 16 de mayo, flexibilizaron determinadas restricciones, permitiéndose, con ciertas limitaciones, la reapertura al público de los hoteles y establecimientos turísticos y de sus zonas comunes. No obstante, las limitaciones a los viajes internacionales y a la movilidad provocaron que la actividad hotelera durante los meses centrales del verano fuera mínima. Además, en las primeras semanas de agosto surgieron rebrotes en los contagios por el virus que condicionaron las reaperturas previstas. A partir de octubre, una nueva ola de contagios obligó a decretar confinamientos y cierres perimetrales, de manera que también en los últimos meses del año la actividad de los alojamientos turísticos fue muy escasa (Exceltur, 2021).

Sobre la base anterior, nos proponemos detectar los cambios que se han producido en el turismo interior en España durante la pandemia de la COVID-19 y discutir el papel que puede desempeñar para facilitar la recuperación turística El resto del artículo se organiza de la siguiente manera. En el próximo apartado se realiza un breve repaso de la literatura. A continuación se aportan algunos datos que justifican la importancia del turismo interior. Después se analiza la información estadística disponible para detectar los cambios que, como consecuencia de la pandemia, ha experimentado el turismo interior en el caso particular de España. Más adelante se analizan las acciones públicas que, a distintos niveles, se han implementado para promocionar el turismo interior. Terminaremos con las conclusiones.

\section{Repaso de la literatura}

Tradicionalmente, el turismo interior ha despertado menos interés que el internacional, quizás debido a que, a diferencia de este último, no proporciona ingresos en divisas (Jafari, 1986). Incluso se ha transmitido la idea de que el turismo interior es el «pariente pobre» frente a un turismo internacional más glamuroso (Scheyvens, 2007). No obstante, a nivel más general, existe evidencia en la literatura académica que demuestra la relevancia del turismo interior para impulsar el crecimiento económico regional y su capacidad para ayudar a resolver algunos de los problemas que ocasiona el turismo internacional (Archer, 1978; Cortés-Jiménez, 2008; Goh et al., 2015; Haddad et al., 2013). Asimismo, los estudios previos han demostrado que el papel que desempeña el turismo nacional adquiere especial protagonismo en tiempos de crisis, ya que, aunque no tenga el mismo impacto positivo en la balanza de pagos que el turismo internacional, se reconoce su potencial para crear renta y empleo (Sheldon y Dwyer, 2010). En este sentido, existen ejemplos de la oportunidad de promocionar el turismo interior ante las crisis económicas (De Sausmarez, 2007; Scheyvens, 2002).

La crisis financiera de 2007 confirmó que, ante las dificultades económicas, los turistas potenciales prefieren destinos que estén próximos a sus lugares de origen (Smeral, 2009), lo que hace pensar en una sustitución de los viajes internacionales por los nacionales. Además, se ha observado que, en determinadas circunstancias, los potenciales turistas dejan de viajar a lugares con dificultades, pero no $D$ 
disminuyen su propensión a viajar (Cooper, 2006).

Respecto a las investigaciones centradas, específicamente, en el caso español, Guardia et al. (2014) demostraron que la demanda interna de servicios turísticos dependía de las características de las comunidades autónomas de origen y destino (población, PIB y distancia). Benítez y Cabrera (2011) aplicaron un análisis shift-share para confirmar, entre otras cosas, que las comunidades autónomas estaban especializadas en el turismo de sus propios residentes y en los de las comunidades vecinas. El trabajo de Arbulú et al. (2021) resulta particularmente interesante, ya que tiene como objetivo evaluar la capacidad del turismo interior español para paliar la crisis provocada por la caída de la demanda internacional como consecuencia de la COVID-19. Sus resultados demostraron que la reorientación del turismo emisor hacia el mercado nacional podría compensar la disminución de la demanda turística internacional, aunque su potencial variaba ampliamente entre regiones, dependiendo, entre otros factores, de la orientación de su tradicional mercado interno.

\section{La importancia del turismo interior}

Las llegadas internacionales en el mundo disminuyeron unos mil millones en 2020, lo que supuso una caída de más del $70 \%$ respecto a 2019 y un retroceso en las cifras absolutas hasta niveles de 1990 (UNWTO, 2020a). En el caso de España, pasó de recibir 83,5 millones de turistas en 2019 a 19 millones en 2020, lo que representó una caída de algo más de $77 \%$ (Gráfico 1). Se presenta, por tanto, la oportunidad de valorar la capacidad del turismo nacional para compensar o aliviar la crisis turística provocada por la COVID-19.

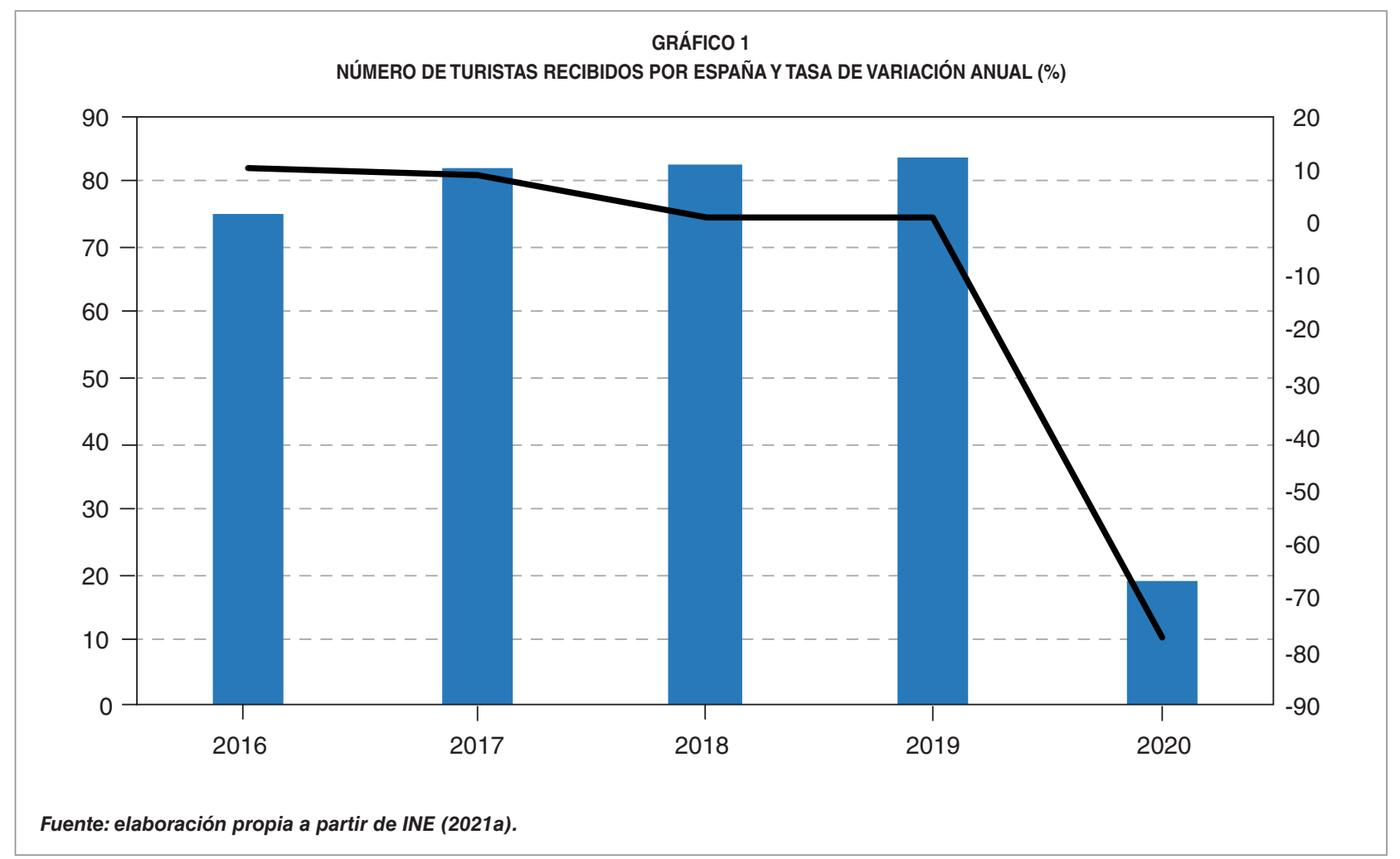


CUADRO 1

INDICADORES DE LA IMPORTANCIA DEL TURISMO INTERIOR EN 2018

\begin{tabular}{|c|c|c|c|c|}
\hline Indicadores & Alemania & Francia & España & Reino Unido \\
\hline \% sobre el total de viajes & 80,3 & 68,1 & 67,2 & 76,8 \\
\hline$\%$ sobre el total de pernoctaciones en hoteles y establecimientos similares ......... & 76,1 & 63,4 & 34,4 & 50,0 \\
\hline$\%$ sobre el total de gasto turístico. & 87,1 & 59,5 & 54,4 & 76,2 \\
\hline Viajes turísticos nacionales per cápita & 1,9 & 2,9 & 3,7 & 1,8 \\
\hline
\end{tabular}

El potencial del turismo interior para superar la crisis turística está basado, en primer término, en su importancia relativa. Según la OMT, el número de viajes que realizan los turistas dentro de su propio país es, a nivel global, más de seis veces mayor que los que realizan los turistas internacionales (UNWTO, 2020b). Lógicamente, la población y el tamaño geográfico están relacionados directamente con la importancia que tiene el turismo interno en cada país. Así, en India, Brasil, China o Estados Unidos los viajes nacionales representan más del $95 \%$ de todos los viajes. Pues bien, la propia OMT incluye a España entre los países que poseen un gran mercado turístico nacional (UNWTO, 2020b).

El Cuadro 1 recoge varios indicadores de la importancia relativa del turismo interior en algunos países europeos de los que se dispone de información. En el caso de España, el turismo nacional representa el $67 \%$ del total de viajes y el $54 \%$ del gasto turístico. No obstante, solo algo más de la tercera parte de las pernoctaciones en hoteles y establecimientos similares se atribuyen al turismo nacional, a diferencia de lo que ocurre en los otros países considerados (Alemania, Francia y Reino Unido), donde este indicador es apreciablemente mayor. Quizás la importancia que tienen en España los alojamientos que no se comercializan en el mercado (por ejemplo, viviendas en propiedad y de amigos y familiares) en el turismo interior ayude a explicar esta evidencia. En cualquier caso, aunque en España se registran valores inferiores a los de otros países que aparecen en el Cuadro 1 en los indicadores presentados, el número de viajes turísticos nacionales per cápita $(3,7)$ es superior al registrado en Alemania $(1,9)$, Francia $(2,9)$ y Reino Unido $(1,8)$.

\section{El turismo interior en España durante la pandemia}

La información que da contenido a este apartado proviene de la Encuesta de Turismo de Residentes (ERT). Esta encuesta la publica el Instituto Nacional de Estadística (INE) desde que, en 2015, asumió las competencias que, previamente, correspondían al Instituto de Estudios Turísticos cuando elaboraba la Estadística de Movimientos Turísticos de los Españoles (FAMILITUR) (Fullea y Fernández, 2021).

El primer dato relevante se refiere a la evolución en el número de pernoctaciones. El Cuadro 2 refleja la caída experimentada en 2020 respecto al año anterior, tanto en las pernoctaciones realizadas en el extranjero como en España. Mientras que en el primer caso la caída fue del $70,4 \%$, en el segundo fue del $30,6 \%$. Esto supuso que el porcentaje de pernoctaciones de los residentes en España en el propio país alcanzara una cifra superior a 91 sobre el total de pernoctaciones en 2020. En consecuencia, la evidencia apunta a que el $D$ 
retroceso en la actividad turística interior ha sido relativamente menor que la experimentada a nivel internacional y que, por tanto, la pandemia ha reforzado la importancia del turismo interior.

Otra información interesante se refiere al porcentaje de pernoctaciones que los residentes realizan en su propia comunidad respecto al total de las realizadas en España. Como demuestra el Cuadro 3, dicho porcentaje ha crecido en 2020 , respecto a lo ocurrido en los años precedentes, en todas las comunidades autónomas.
De hecho, salvo en el caso de Navarra, el porcentaje en 2020 ha sido superior a cualquiera de los registrados en los cinco años anteriores. A partir de ahí podríamos afirmar que una buena parte de los residentes en España no solo ha sustituido los viajes internacionales por los nacionales, sino que ha realizado su actividad turística dentro de su región en vez de fuera de su comunidad.

También resultan destacables los cambios de tendencia que se constatan en el Cuadro 4. $\triangleright$

CUADRO 2

PERNOCTACIONES DE RESIDENTES DE ESPAÑA (2015-2020)

\begin{tabular}{|c|c|c|c|c|c|}
\hline \multirow{2}{*}{ Año } & \multicolumn{2}{|r|}{ Extranjero } & \multicolumn{2}{|r|}{ España } & \multirow{2}{*}{ \% pernoctaciones en España } \\
\hline & Millones & Variación porcentual & Millones & Variación porcentual & \\
\hline 2015 & 133,2 & - & 616,5 & - & 82,2 \\
\hline 2016 & 137,3 & 3,1 & 643,1 & 4,3 & 82,4 \\
\hline 2017 & 141,2 & 2,8 & 660,8 & 2,8 & 82,4 \\
\hline 2018 & 151,8 & 7,5 & 644,4 & $-2,5$ & 80,9 \\
\hline 2019 & 144,4 & $-4,9$ & 649,5 & 0,8 & 81,8 \\
\hline 2020 & 42,7 & $-70,4$ & 450,7 & $-30,6$ & 91,3 \\
\hline
\end{tabular}

CUADRO 3

PORCENTAJES DE PERNOCTACIONES EN LA COMUNIDAD AUTÓNOMA DE RESIDENCIA (2015-2020)

\begin{tabular}{|c|c|c|c|c|c|c|c|c|}
\hline Comunidad autónoma & 2015 & 2016 & 2017 & 2018 & 2019 & Promedio 2015-2019 [1] & 2020 [2] & {$[2]-[1]$} \\
\hline Andalucía .. & 73,3 & 73,4 & 73,3 & 73,4 & 74,0 & 73,5 & 82,3 & 8,8 \\
\hline Aragón ... & 43,1 & 44,3 & 41,6 & 41,9 & 39,1 & 42,0 & 45,6 & 3,6 \\
\hline Asturias .. & 39,2 & 32,9 & 39,8 & 34,7 & 38,7 & 37,1 & 47,9 & 10,8 \\
\hline Baleares & 42,1 & 34,4 & 41,5 & 40,8 & 39,2 & 39,6 & 48,4 & 8,7 \\
\hline Canarias ............ & 62,1 & 64,0 & 70,1 & 66,4 & 63,9 & 65,3 & 78,4 & 13,1 \\
\hline Cantabria & 26,1 & 26,6 & 28,5 & 27,9 & 29,8 & 27,8 & 41,2 & 13,4 \\
\hline Castilla y León & 42,2 & 43,2 & 41,1 & 37,9 & 44,7 & 41,8 & 52,3 & 10,5 \\
\hline Castilla-La Mancha .. & 31,5 & 29,5 & 31,1 & 29,9 & 25,0 & 29,4 & 39,9 & 10,5 \\
\hline Cataluña & 53,4 & 52,4 & 56,1 & 57,0 & 55,0 & 54,8 & 60,5 & 5,7 \\
\hline Comunidad Valenciana ... & 51,9 & 51,2 & 54,0 & 54,4 & 57,9 & 53,8 & 65,1 & 11,3 \\
\hline Extremadura & 38,6 & 38,3 & 39,0 & 40,9 & 36,1 & 38,6 & 48,7 & 10,1 \\
\hline Galicia & 66,5 & 65,7 & 66,1 & 60,7 & 65,6 & 64,9 & 78,8 & 13,9 \\
\hline Madrid & 8,2 & 8,5 & 6,9 & 7,7 & 7,2 & 7,7 & 8,8 & 1,1 \\
\hline Murcia & 36,9 & 37,0 & 43,4 & 42,6 & 41,0 & 40,2 & 46,3 & 6,1 \\
\hline Navarra & 29,0 & 28,9 & 28,0 & 25,1 & 22,3 & 26,7 & 28,0 & 1,3 \\
\hline País Vasco ... & 11,8 & 13,3 & 11,7 & 12,6 & 13,9 & 12,7 & 19,5 & 6,8 \\
\hline La Rioja & 26,2 & 27,6 & 23,1 & 22,2 & 21,1 & 24,0 & 30,0 & 5,9 \\
\hline
\end{tabular}


En primer lugar, se aprecia el mayor peso que han adquirido los alojamientos que no se comercializan en el mercado, es decir, los que se corresponden, básicamente, con la vivienda en propiedad o con la de familiares y amigos. Parece lógico pensar que las restricciones impuestas a la actividad de los alojamientos turísticos de mercado (hoteles y similares) han influido en este resultado, aunque tampoco habría que descartar que los cambios en la propia demanda turística, como consecuencia de la pandemia, hayan alterado las preferencias de los turistas. En cualquier caso, en 2020 , casi el $70 \%$ de las pernoctaciones fueron en alojamientos «no de mercado», mientras que el promedio de los cinco años anteriores no superó el $62 \%$.

En cuanto al método de transporte se observa un importante crecimiento del vehículo propio frente a otros medios (avión, ferrocarril, etcétera). Una vez más, la combinación de los shocks de oferta y demanda podría explicar el crecimiento de casi 11 puntos en 2020 , frente a la media de lo ocurrido entre 2015 y 2019, en el uso del vehículo propio para los desplazamientos. En el año 2020, el 79,1\% de los residentes en España utilizó el vehículo propio para desplazarse a un alojamiento distinto al habitual.

La forma de organización del viaje de los residentes en España ha sido, en los años previos a la pandemia, en un porcentaje cercano a 95, sin paquete turístico. Pues bien, en el año 2020 esa cifra aumentó hasta alcanzar el $98,4 \%$.

Respecto a la duración del viaje, se produjo un destacable incremento en 2020 de aquellos que han supuesto más de quince pernoctaciones. Así, mientras que el promedio entre 2015 y 2019 de los viajes con esas pernoctaciones fue de $26,1 \%$, en 2020 supusieron el $36,8 \%$.

Por último, como refleja el Cuadro 5 , el gasto medio por persona es sensiblemente superior para los residentes que viajan al extranjero que para los que lo hacen dentro de España. No obstante, mientras que en 2020 dicho gasto disminuyó respecto a los años anteriores, ocurrió lo contrario tanto para el gasto medio dentro de la comunidad autónoma de residencia del viajero como fuera de la misma. Lo cual es compatible con el hecho de que los residentes en España sustituyeran el turismo internacional por el interior.

CUADRO 4

PORCENTAJE SOBRE EL TOTAL DE PERNOCTACIONES (2015-2020)

\begin{tabular}{|c|c|c|c|c|c|c|c|c|}
\hline & 2015 & 2016 & 2017 & 2018 & 2019 & Promedio 2015-2019 [1] & 2020 [2] & [2]-[1] \\
\hline No mercado ... & 63,2 & 63,3 & 62,0 & 60,5 & 59,6 & 61,7 & 69,6 & 7,9 \\
\hline Vehículo propio & 68,2 & 68,1 & 69,2 & 68,0 & 67,5 & 68,2 & 79,1 & 10,9 \\
\hline Sin paquete turístico & 94,7 & 94,9 & 94,6 & 94,4 & 94,6 & 94,6 & 98,4 & 3,8 \\
\hline Más de 15 noches ... & 27,9 & 27,7 & 26,7 & 24,2 & 24,1 & 26,1 & 36,8 & 10,7 \\
\hline
\end{tabular}

CUADRO 5

GASTO MEDIO POR PERSONA (euros)

\begin{tabular}{|c|c|c|c|c|c|c|c|c|}
\hline & 2015 & 2016 & 2017 & 2018 & 2019 & Promedio 2015-2019 [1] & $2020[2]$ & [2]-[1] \\
\hline Extranjero & 788,1 & 841,2 & 825,7 & 832,9 & 797,8 & 817,1 & 704,8 & $-112,3$ \\
\hline Dentro de comunidad autónoma ... & 90,8 & 92,7 & 93,0 & 97,5 & 105,3 & 95,9 & 113,5 & 17,6 \\
\hline A otra comunidad autónoma & 239,4 & 251,7 & 255,1 & 260,2 & 270,4 & 255,4 & 283,3 & 27,9 \\
\hline
\end{tabular}




\section{La promoción de turismo interior}

De acuerdo con lo expuesto, los datos confirman que, durante la pandemia, el turismo interior ha reforzado su importancia respecto al turismo internacional, y ha intensificado, al menos en España, algunas de las tendencias que habían caracterizado su evolución en los últimos años. En estas circunstancias, muchos Gobiernos han adoptado medidas para promocionar el turismo interior. De acuerdo con la OMT, el objetivo de estas iniciativas ha sido, de entrada, restaurar la confianza en el sector turístico para, posteriormente, avanzar en las iniciativas para atraer a los turistas internacionales (UNWTO, 2020b). Al margen del apoyo financiero que, con carácter transversal, se ha implementado para garantizar la liquidez de las empresas, especialmente dirigido a las pequeñas y medianas, las medidas adoptadas para la promoción del turismo interior se podrían agrupar en seis categorías (UNWTO, 2020b):

1. Incentivos financieros. Aquí se incluyen las reducciones del IVA para los vuelos nacionales y determinados servicios turísticos, los bonos que ofrecen descuentos en establecimientos turísticos y los long weekends, que consisten en trasladar días festivos nacionales para ampliar los fines de semana y, de esta manera, hacer más atractivos los viajes interiores.

2. Campañas de marketing. En algunos casos se han reforzado las campañas existentes y, en otros, además, se han impulsado otras nuevas que, en ocasiones, se han aprovechado para promocionar destinos menos conocidos $y$ áreas rurales o naturales, con el objetivo de estimular la demanda turística interna y, al mismo tiempo, generar renta y empleo en los lugares que más lo necesitaban.

3. Desarrollo de productos. Se trata de ofrecer actividades integradas que animen a los turistas a viajar dentro del país explotando sus motivaciones hacia la cultura, la gastronomía, la aventura, etc. También se han promocionado viajes por carretera que conectan diferentes destinos nacionales.

4. Alianzas público-privadas. Además de estrechar los vínculos entre los distintos organismos y niveles de la Administración pública, se han impulsado las relaciones entre los ámbitos públicos y privados para la promoción de ofertas especiales o para facilitar el acceso al mercado de las empresas turísticas a través de ferias o plataformas digitales para estimular el turismo interior.

5. Inteligencia de mercado. Esta herramienta se ha utilizado al recopilar datos, frecuentemente a través de encuestas sobre las preferencias y el comportamiento del viajero nacional, para el diseño de estrategias orientadas a la promoción del turismo interno o para el desarrollo de nuevos productos turísticos.

6. Capacitación y formación. Se han aprovechado las posibilidades que ofrece la conexión on line para mejorar las habilidades profesionales de los trabajadores que están en contacto directo con los turistas nacionales y para fortalecer la cadena de comercialización del turismo interior.

Por su parte, el Gobierno español presentó en junio de 2020 el Plan de Impulso para el $\triangleright$ 
Sector Turístico: Hacia un Turismo Seguro y Sostenible (en adelante el Plan) (Gobierno de España, 2020) con una dotación económica de 4.262,8 millones de euros, los cuales se orientan, básicamente, a las empresas turísticas, es decir, al lado de la oferta. Así, 3.256 millones de euros, es decir, algo más del $76 \%$ del total, se dedica a mantener la liquidez y la solvencia empresarial, que incluye una línea de avales del Instituto de Crédito Oficial (ICO). Además, se destinan otros 515 millones de euros, que representan un $12 \%$ de la dotación financiera del Plan, al denominado Fondo Financiero del Estado para la Competitividad Turística (FOCIT) que consiste en préstamos a proyectos presentados por empresas turísticas relacionados, especialmente, con la sostenibilidad y la digitalización.

No obstante, aunque el Plan, al menos en términos financieros, está orientado a las empresas turísticas, también incluye referencias a la demanda. Ahora bien, se observa un cierto sesgo hacia la demanda turística internacional. Por ejemplo, se crea una línea de préstamos de 216 millones de euros con tipo de interés bonificado para financiar proyectos empresariales entre los que se encuentran los «orientados a la internacionalización de los destinos y empresas turísticas». Asimismo, el denominado Programa de Planes de Sostenibilidad Turística en Destinos, dotado con 53 millones de euros, tiene, entre otros objetivos, desarrollar «productos atractivos, seguros y saludables para la demanda internacional (turismo cultural, turismo activo, ecoturismo, turismo enogastronómico, turismo industrial o, entre otros, el turismo científico)». También se habla de un Modelo de Conocimiento Integral, que sería la base de un «nuevo observatorio de inteligencia turística» que «incluye la generación de inteligencia de mercados y el seguimiento y análisis de la situación de la demanda internacional». Por último, la puesta en marcha de un Plan de Marketing 2020-2024, al que se destinan 33,3 millones de euros, está pensado para «reactivar de forma rápida el turismo internacional hacia España».

Las alusiones al turismo interior o nacional son menos numerosas en el Plan y, con frecuencia, aparecen junto a una referencia al turismo internacional. Así, por ejemplo, ocurre cuando se menciona la creación del distintivo Safe Tourism para «reposicionarse como destino turístico seguro en los mercados nacional e internacional», o cuando se establece el refuerzo de la «red de destinos turísticos inteligentes [...] en el ámbito nacional e internacional». En cualquier caso, el Plan incluye una referencia específica al turismo interior que resulta especialmente significativa. En efecto, en el apartado dedicado a la «mejora del conocimiento integral» se afirma lo siguiente:

«La Administración turística del Estado debe liderar [...] el análisis de la coyuntura turística que ponga el foco en el turismo doméstico, excursionistas y turistas nacionales, que serán los que van a protagonizar, no sólo los primeros momentos de reactivación de la actividad turística pos-COVID-19, sino buena parte del crecimiento posterior de la misma. [...] será fundamental poder realizar un seguimiento continuo del ritmo de recuperación de la demanda doméstica en las diferentes regiones, y de aquella que, aunque a pequeña escala, pudiera comenzar a producirse en un segundo momento a nivel internacional». (Gobierno de España, 2020)

Parece, por tanto, que se confía en el turismo nacional para iniciar la recuperación que $D$ 
se confirmaría, posteriormente, con el incremento de la demanda turística internacional.

Además de las acciones implementadas por el Gobierno español, las Administraciones regionales y locales han tomado iniciativas específicas destinadas al sector turístico. Entre ellas merecen especial atención los bonos turísticos que han creado un conjunto muy amplio de comunidades autónomas con el objetivo de promocionar el turismo interior para reactivar la actividad turística dentro de cada región. Sus características son diversas, aunque, normalmente, consisten en descuentos para estancias en hoteles en la propia comunidad (Tourinews, 2021).

En la comunicación COM(2020) 550 final de la Comisión Europea se mencionaban los bonos como un instrumento de promoción del turismo local y se afirmaba que en los Estados miembros en los que se han establecido «los clientes se han mostrado entusiasmados ante la idea de apoyar a sus hoteles o restaurantes favoritos». Aunque también han recibido críticas. Así, se ha dudado de su capacidad para estimular los viajes al beneficiar a personas que hubieran viajado, aunque no hubieran recibido el bono, y se ha señalado un supuesto efecto regresivo al no distinguir en función de la renta del receptor del bono (Jiménez, 2021).

En cualquier caso, parece evidente que, en el caso de España, la asunción de competencias sobre la promoción turística por los distintos niveles de la Administración pública puede generar cierta discusión. Es posible, por ejemplo, que la competencia entre regiones por atraer o retener a los turistas desemboque en un juego de suma cero, o que exista cierto margen de mejora en las campañas de promoción turística a través de la coordinación entre regiones $\mathrm{y}$, especialmente, entre las que son vecinas.

\section{Conclusiones}

La pandemia de la COVID-19 ha provocado shocks de oferta y demanda en el mercado turístico que han generado una crisis turística muy grave. En este contexto, se ha planteado la oportunidad de promocionar el turismo interno para tratar de compensar, en cierta medida, la caída de la demanda turística internacional. De hecho, la investigación académica había demostrado, hace tiempo, la capacidad que tiene el turismo interior para generar renta y empleo, y para contribuir a la convergencia regional. Más recientemente, se ha acreditado su potencial para paliar la disminución de la demanda turística internacional causada por la pandemia. Además, los datos demuestran que el turismo interior genera, en muchos países, la mayor parte de la actividad turística.

En el caso de España, el análisis de la información estadística disponible confirma que durante la pandemia se ha reforzado la importancia del turismo interior, lo que sugiere que se han sustituido los viajes internacionales por los nacionales $y$, especialmente, por los realizados dentro de la propia comunidad autónoma de residencia. Otras características del turismo interior que se han reforzado durante la pandemia han sido las pernoctaciones en alojamientos que no se comercializan en el mercado, el uso del vehículo particular para los viajes, el carácter marginal de los paquetes turísticos como forma de organización de las vacaciones y el incremento de la duración de la estancia en el destino.

En cuanto a las medidas implementadas por los Gobiernos para promocionar el turismo interior, en el caso de España con la aprobación del denominado Plan de Impulso para el Sector Turístico: hacia un Turismo Seguro y Sostenible, se ha confirmado la importancia de las medidas financieras para facilitar el acceso $\square$ 
al crédito por parte de las empresas. En este sentido, el Plan está orientado, fundamentalmente, hacia la oferta turística. Respecto a la demanda, se dedica una especial atención a la demanda turística internacional y se plantea la posibilidad de que el turismo interior inicie la recuperación que se consolidaría posteriormente con el aumento de la demanda turística internacional. Paralelamente, y con independencia del mencionado Plan, las Administraciones públicas regionales y locales han tomado sus propias iniciativas, como los bonos turísticos, con el objetivo de promocionar el turismo dentro de sus propias comunidades.

En definitiva, dentro del actual debate sobre la configuración del sector turístico que ha surgido como consecuencia de la crisis de la pandemia, parece pertinente incorporar a la discusión el papel que puede jugar el turismo interior, no solo como detonante de la recuperación, sino como base del desarrollo equilibrado y sostenible que se pretende conseguir.

\section{Bibliografía}

Arbulú, I., Razumova, M., Rey-Maquieira, J., y Sastre, F. (2021). Can domestic tourism relieve the COVID-19 tourist industry crisis? The case of Spain. Journal of Destination Marketing \& Management, 20. https://doi.org/10.1016/j.jdmm. 2021.100568

Archer, B. (1978). Domestic tourism as a development factor. Annals of Tourism Research, 5(1), 126-141. https://doi.org/10.1016/0160-7383(78)90007-5

Benítez, J. J., y Cabrera, S. (2011). Análisis del turismo nacional en las comunidades autónomas peninsulares españolas: especialización y ventaja competitiva. Investigaciones Regionales, 20, 29-49. https://ebuah.uah.es/xmlui/bitstream/ handle/10017/28698/analisis_benitez_IR_2011_ N20.pdf? sequence $=1$ \&isAllowed $=y$
Blake, A., y Sinclair, M. T. (2003). Tourism crisis management: US response to September 11. Annals of Tourism Research, 30(4), 813-832. https://doi.org/10.1016/S0160-7383(03)00056-2

Comisión Europea. (2020). Comunicación (Com (220) 550 final) de 13 de mayo de 2020, de la Comisión al Parlamento Europeo, al Consejo, al Comité Económico y Social Europeo y al Comité de las Regiones. El turismo y el transporte en 2020 y en adelante. https://ec.europa.eu/info/sites/default/files/communication-commissiontourism-transport-2020-and-beyond_es.pdf

Cooper, M. (2006). Japanese tourism and the SARS epidemic of 2003. Journal of Travel \& Tourism Marketing, 19(2-3), 117-131. https://doi.org/ 10.1300/J073v19n02_10

Cortés-Jiménez, I. (2008). Which type of tourism matters to the regional economic growth? The cases of Spain and Italy. International Journal of Tourism Research, 10(2), 127-139. https://doi. org/10.1002/jtr.646

De Sausmarez, N. (2007). The potential for tourism in post-crisis recovery: Lessons from Malaysia's experience of the Asian financial crisis. Asia Pacific Business Review, 13(2), 277-299. https:// doi.org/10.1080/13602380601045587

Exceltur. (2021). Barómetro de la rentabilidad de los destinos turísticos españoles. Exceltur, Alianza para la excelencia turística (32). https://www. exceltur.org/wp-content/uploads/2021/02/Baro\% CC\%81metro-Rentabilidad-y-el-Empleo-EneroDiciembre-2020.pdf

Fullea, C., y Fernández, A. (2021). La Encuesta de Turismo de Residentes (ETR/FAMILITUR). Revista de Estadística y Sociedad (80), 21-25. http://www.revistaindice.com/numero80/p21.pdf

Gobierno de España. (2020). Plan de impulso para el sector turístico: hacia un turismo seguro y sostenible. https://www.lamoncloa.gob.es/serviciosdeprensa/notasprensa/industria/Documents/2020/20062020_PlanTurismo.pdf

Goh, C., Li, H., y Zhang, Q. (2015). Achieving balanced regional development in China: is $D$ 
domestic or international tourism more efficacious? Tourism Economics, 21(2), 369-386. https://doi. org/10.5367/te.2013.0353

Guardia, T., Muro, J., y Such, M. J. (2014). Measuring and analysing domestic tourism: the importance of an origin and destination matrix. Tourism Economics, 20(3), 451-472. https://doi. org/10.5367/te.2013.0286

Haddad, E. A., Porsse, A. A., y Rabahy, W. (2013). Domestic tourism and regional inequality in Brazil. Tourism Economics, 19(1), 173-186. https://doi.org/10.5367/te.2013.0185

INE. (2021a). Estadística de movimientos turísticos en frontera. Frontur. https://ine.es/dyngs/INEbase/es/operacion.htm?c=Estadistica_C\&cid= $1254736176996 \&$ menu $=u$ ltiDatos $\& i d p=$ 1254735576863

INE. (2021b). Encuesta de turismo de residentes. ETR/Familitur. Primer trimestre 2021. https://www. ine.es/dyngs/INEbase/es/operacion.htm?c= Estadistica_C\&cid=1254736176990\&menu=ultiDatos\&idp $=1254735576863$

Jafari, J. (1986). On domestic tourism. Annals of Tourism Research, 13(3), 491-496. https://doi. org/10.1016/0160-7383(86)90033-2

Jiménez, J. L. (9 de junio de 2021). Bonos turísticos (o cómo malgastar dinero público). Nada es gratis. https://nadaesgratis.es/juan-luis-jimenez/bonosturisticos-o-como-malgastar-dinero-publico

McKercher, B., y Chon, K. (2004). The over-reaction to SARS and the collapse of Asian tourism. Annals of Tourism Research, 31(3), 716-719. https://doi.org/10.1016/j.annals.2003.11.002

Pechlaner, H., y Frehse, J. (2010). Financial crisis and tourism. In R. Conrady y M. Buck, Trends and Issues in Global Tourism 2010 (pp. 27-38). Springer. https://link.springer.com/chapter/10. 1007/978-3-642-10829-7_4

Ritchie, B. W., y Jiang, Y. (2019). A review of research on tourism risk, crisis and disaster management: Launching the annals of tourism research curated collection on tourism risk, crisis and disaster management. Annals of Tourism Research, 79. https://doi.org/10.1016/j.annals.2019. 102812

Scheyvens, R. (2002). Tourism for development: empowering communities. Prentice Hall.

Scheyvens, R. (2007). Poor cousins no more: valuing the development potential of domestic and diaspora tourism. Progress in Development Studies, 7(4), 307-325. https://doi.org/10.1177/ 146499340700700403

Sheldon, P., y Dwyer, L. (2010). The global financial crisis and tourism: Perspectives of the academy. Journal of Travel Research, 49(1), 3-4. https://doi.org/10.1177/0047287509353191

Škare, M., Ribeiro, D. E., y Porada-Rochoń, M. (2021). Impact of COVID-19 on the travel and tourism industry. Technological Forecasting and Social Change, 163. https://doi.org/10.1016/j. techfore.2020.120469

Smeral, E. (2009). The impact of the financial and economic crisis on European tourism. Journal of Travel Research, 48(1), 3-13. https://doi.org/ $10.1177 / 0047287509336332$

Tourinews. (5 de junio de 2021). ¿Qué comunidades autónomas han puesto en marcha el 'Bono Turístico'? https://www.tourinews.es/espana/bono-turistico-comunidades-autonomas-resumen_4464531_102.html

UNWTO. (2020a). Tourism back to 1990 levels as arrivals fall by more than $70 \%$. https://www. unwto.org/news/tourism-back-to-1990-levelsas-arrivals-fall-by-more-than-70

UNWTO. (2020b). UNWTO briefing note - Tourism and COVID-19, Issue 3. Understanding domestic tourism and seizing its opportunities. Madrid: UNWTO.https://doi.org/10.18111/9789284422111

UNWTO. (2021). 2020: Worst year in tourism history with 1 billion fewer international arrivals. https:// www. unwto.org/news/2020-worst-year-intourism-history-with-1-billion-fewer-internationalarrivals 
